\title{
ERS International Congress 2020: highlights from the Paediatric Assembly
}

\author{
Cristina Ardura-Garcia (1) 1 , Sara Cuevas-Ocaña², Nadine Freitag ${ }^{3}$, \\ Asterios Kampouras $\mathbb{1}^{4}$, John A. King ${ }^{5}$, Panayiotis Kouis $\mathbb{1}^{6}$, \\ Sara M. Mensink-Bout ${ }^{7}$, Abigail L. Whitehouse ${ }^{8}$, Emma Williams ${ }^{9}$, \\ Theodore Dassios ${ }^{9}$, Liesbeth Duijts (10 ${ }^{10}$, Refika H Ersu ${ }^{11}$, Erol A. Gaillard ${ }^{12}$, \\ Alex R. Horsley $\mathbb{1}^{13}$, Marijke Proesmans ${ }^{14}$, Robbert Rottier (15) ${ }^{15,16}$, \\ Dirk Schramm ${ }^{3}$, Alexander Moeller $\circledast^{17}$ and Marielle W. Pijnenburg ${ }^{18}$
}

ABSTRACT In this review, the Paediatric Assembly of the European Respiratory Society (ERS) presents a summary of the highlights and most relevant findings in the field of paediatric respiratory medicine presented at the virtual ERS International Congress 2020. Early Career Members of the ERS and Chairs of the different Groups comprising the Paediatric Assembly discuss a selection of the presented research. These cover a wide range of research areas, including respiratory physiology and sleep, asthma and allergy, cystic fibrosis, respiratory infection and immunology, neonatology and intensive care, epidemiology, bronchology and lung and airway development. Specifically, we describe the long-term effect in lung function of premature birth, mode of delivery and chronic respiratory conditions such as cystic fibrosis. In paediatric asthma, we present risk factors, phenotypes and their progression with age, and the challenges in diagnosis. We confirm the value of the lung clearance index to detect early lung changes in cystic fibrosis. For bronchiectasis treatment, we highlight the importance of identifying treatable traits. The use of biomarkers and genotypes to identify infants at risk of long-term respiratory morbidity is also discussed. We present the long-term impact on respiratory health of early life and fetal exposures to maternal obesity and intrauterine hypoxia, mechanical ventilation hyperoxia, aeroallergens, air pollution, vitamin A deficient intake and bronchitis. Moreover, we report on the use of metabolomics and genetic analysis to understand the effect of these exposures on lung growth and alveolar development. Finally, we stress the need to establish multidisciplinary teams to treat complex airway pathologies.

@ERSpublications

Highlights from the Paediatrics Assembly at the \#ERSCongress 2020 https://bit.ly/3ptcnFr

Cite this article as: Ardura-Garcia C, Cuevas-Ocaña S, Freitag N, et al. ERS International Congress 2020: highlights from the Paediatric Assembly. ERJ Open Res 2021; 7: 00893-2020 [https://doi.org/ 10.1183/23120541.00893-2020].
Received: 30 Nov 2020 | Accepted: 24 Jan 2021

( T) The authors 2021. This version is distributed under the terms of the Creative Commons Attribution Non-Commercial Licence 4.0. For commercial reproduction rights and permissions contact permissions@ersnet.org 
The Paediatric Assembly (Assembly 7) reviewed the latest paediatric respiratory research presented by leading experts and early career members at the European Respiratory Society (ERS) virtual International Congress 2020. More than 290 abstracts were presented by Assembly 7 members in several oral and e-poster sessions. Assembly 7 also organised nine sessions including scientific symposia, Hot Topics, Expert View, Grand Round, "Lungs on fire", State of the Art and challenging clinical cases sessions, and the Scientific Year in Review. With significant input from our Early Career Members, we here review some of the major paediatric highlights from the ERS 2020 virtual International Congress 2020. The specific sessions were selected by the Chairs of each of the eight groups that compose the Paediatric Assembly and include oral and poster sessions, Hot Topics sessions, symposia and a live procedure.

\section{Paediatric respiratory physiology and sleep}

Although neonatal care has evolved dramatically in the last 30 years, few data exist on respiratory function of preterm neonates in adult life. This was the focus of several studies in the oral presentations session of the paediatric respiratory physiology and sleep group. BÅRDSEN et al. [1] studied three population-based cohorts (1982-1985, 1991-1992 and 1999-2000) of young adults born extremely preterm (gestational age $<28$ weeks) and compared them with adults born term by performing spirometry at the age of 18 years. Subjects born extremely preterm had significantly lower forced expiratory volume in $1 \mathrm{~s}\left(\mathrm{FEV}_{1}\right) \mathrm{z}$-scores at the age of 18 years; however, significant improvement of the respiratory function of the subjects born extremely preterm was noted over the three decades. MARsol et al. [2] investigated lung function trajectories of 109 children born preterm with body plethysmography, bronchodilator test and spirometry at the ages of 4, 5 and 6 years and compared these with lung function of children born at term. Even though lung function in subjects born prematurely was lower at birth, no differences were noted during preschool years, suggesting a catch-up of lung function in this group of children born prematurely. Mahmoud et al. [3] investigated whether mode of delivery can influence respiratory outcomes during the first year of life. In this study, 580 full-term infants born by caesarean section (140 babies) or vaginal delivery (440 babies) performed multiple breath washout (MBW) measurements, tidal breathing measurements and were monitored for respiratory rate and symptoms during the first year of life. Children born by caesarean section did not have higher rates of respiratory symptoms in the first year of life. There were no differences in MBW or overall lung function between the two groups either.

During this same oral session, FouzAs et al. [4] presented their findings on the pattern of FEV $_{1}$ variability in healthy children and adolescents. In total, 92 children aged 6-18 years performed spirometry twice per day for 3 months. They showed that as age increased, the detrended fluctuation analysis of lung function (DFA) $\alpha$ exponent, an index of a person's lung function variability, decreased, and sample entropy of $\mathrm{FEV}_{1}$ was elevated. These findings point to an increased adaptability of the respiratory system especially through adolescence. KENTGENs et al. [5] investigated whether volumetric capnography (VCap) can reflect ventilation inhomogeneity. In their study, MBW and VCap measurements from 63 healthy children and 50 children with cystic fibrosis (mean age 9.9 years) were evaluated. They found that obtaining capnographic indices (CII) derived by VCap is feasible, and CII showed a significant correlation with lung clearance index (LCI) in children with cystic fibrosis $\left(\mathrm{r}^{2}=0.42 ; \mathrm{p}<0.001\right)$. Thus, VCap seems promising as an indicator of ventilation inhomogeneity but further studies are needed to reveal its minimal methodological requirements along with its true clinical value.

\footnotetext{
Affiliations: 'Institute of Social and Preventive Medicine, University of Bern, Bern, Switzerland. ${ }^{2}$ Division of Cancer and Stem Cells, Biodiscovery Institute, School of Medicine, University of Nottingham, University Park, Nottingham, UK. ${ }^{3}$ Dept of General Paediatrics, Neonatology and Paediatric Cardiology, University Childreńs Hospital, Heinrich-Heine-University, Duesseldorf, Germany. ${ }^{4}$ Paediatric Dept, 424 General Military Hospital, Thessaloniki, Greece. ${ }^{5}$ Dept of Paediatric Respiratory Medicine, Royal Brompton Hospital, London, UK. ${ }^{6}$ Respiratory Physiology Laboratory, Medical School, University of Cyprus, Nicosia, Cyprus. ${ }^{7}$ Dept of Paediatrics, Division of Respiratory Medicine and Allergology, Erasmus MC, University Medical Centre, Rotterdam, The Netherlands. ${ }^{8}$ Centre for Genomics and Child Health, Blizard Institute, Queen Mary University of London, London, UK. 'Women and Children's Health, School of Life course Sciences, Faculty of Life Sciences and Medicine, King's College London, London, UK. ${ }^{10}$ Dept of Paediatrics, Division of Neonatology, Erasmus MC, University Medical Centre, Rotterdam, The Netherlands. ${ }^{11}$ Division of Respirology, University of Ottawa Children's Hospital of Eastern Ontario, Ottawa, Canada. ${ }^{12}$ Department of Respiratory Sciences, University of Leicester, Leicester, UK. ${ }^{13}$ Division of Infection, Immunity and Respiratory Medicine, University of Manchester, Manchester, UK. ${ }^{14}$ Dept of Paediatrics, University Hospitals Leuven, Leuven, Belgium. ${ }^{15}$ Dept of Paediatric Surgery, Erasmus Medical Centre - Sophia Children's Hospital, Rotterdam, The Netherlands. ${ }^{16}$ Dept of Cell Biology, Erasmus MC, Rotterdam, the Netherlands. ${ }^{17}$ Division of Paediatric Pulmonology, University Children's Hospital Zurich, University of Zurich, Zurich, Switzerland. ${ }^{18}$ Department of Paediatrics, Division of Paediatric Respiratory Medicine and Allergology, Erasmus MC, University Medical Centre, Rotterdam, The Netherlands.
}

Correspondence: Cristina Ardura-Garcia, Institute of Social and Preventive Medicine, University of Bern, Mittelstrasse 43, 3012 Bern, Switzerland. E-mail: cristina.ardurađispm.unibe.ch 
In the field of paediatric sleep medicine, obesity and obstructive sleep apnoea (OSA) have been shown to be associated with multifaceted derangements in metabolic and cardiovascular function, including endothelial dysfunction. Mylemans et al. [6], examined whether weight loss can have an effect on endothelial function and OSA in children with obesity and presented their findings during the oral presentation session. After overweight subjects lost weight, their endothelial function improved, with smaller improvements seen in the OSA subgroup, suggesting both OSA and obesity independently increase the risk for endothelial dysfunction in children.

In the poster session of the Paediatric Respiratory Physiology and Sleep Group, Hatziagorou et al. [7] showed the course of deterioration of LCI in cystic fibrosis patients from childhood to adulthood. In the field of congenital lung malformations MACLEOD et al. [8] presented evidence showing that early surgical treatment did not seem to be a significant influencing factor for long-term respiratory function. Many studies also focussed on sleep disordered breathing (SDB) in the poster session. Of note, EraLP et al. [9] found that a single polygraphy can be used as an adequate measure for paediatric OSA. LeLLi et al. [10] noted that the prevalence of SDB in children with chronic kidney disease was 10-times higher than in the general paediatric population while BEYDON et al. [11] calculated the cut-offs for Snoring Index (SI) linked to OSA, and found SI cut-offs of $172 \mathrm{~h}^{-1}$ and $267 \mathrm{~h}^{-1}$ to be linked to non-severe and severe OSA, respectively.

In summary, this year's novel research in paediatric physiology and sleep explored the long-term lung function in children born prematurely and children with cystic fibrosis. Influence of mode of delivery and surgical treatment on lung function was also studied. Additionally, researchers highlighted the variability and adaptability of the respiratory system throughout childhood. Research supported the use of alternative diagnostic methods for OSA and also investigators showed that obesity and OSA can independently increase the risk of adverse health effects.

\section{Paediatric asthma and allergy}

The main themes that emerged in the "Hot Topics in Paediatric Asthma" session, included challenges in asthma diagnosis, asthma phenotypes and their progression with age, risk factors for developing asthma and asthma prevalence in primary care.

Starting with risk factors for asthma, SøRENSEN et al. [12] identified cases of severe bronchiolitis during infancy and age-matched controls to examine the link with asthma at the age of 19 years. Current asthma was more prevalent in the post-bronchiolitis group (24\% versus $12 \% ; \mathrm{p}=0.005)$ and $\mathrm{z}$-scores for $\mathrm{FEV}_{1}$, forced expiratory flow at $25-75 \%$ of forced vital capacity $(\mathrm{FVC})\left(\mathrm{FEF}_{25-75 \%}\right)$ and $\mathrm{FEV}_{1} / \mathrm{FVC}$ ratio were significantly lower $(\mathrm{p}=0.003,0.001$ and 0.002 , respectively). There was no difference in atopy between the two groups (positive skin prick test (SPT) or specific immunoglobulin (Ig)E $>0.35 \mathrm{kU} \cdot \mathrm{L}^{-1}$ ). Lung function in early childhood was not measured; therefore, we do not know whether genetically determined small airways have contributed to these findings.

Moving on to asthma phenotyping, Hose et al. [13] used patient questionnaires over 2 years to define asthma phenotypes in children as part of the All Age Asthma Cohort (ALLIANCE) cohort in Germany. Latent class analysis identified six latent classes (LC) as the best fit model. LC1 was characterised by persistent symptoms but low atopy, LC2 by atopy and poor control and LC3 by atopy and good control. LC4 had poor control but strong environmental trigger exposures including maternal smoking in pregnancy. LC5 tended to be pre-school children in whom severe symptoms remitted after the first hospitalisation and LC6 consisted of well-controlled, non-atopic children. When comparing classes with LC6 (good control, non-atopic), over time, LC4 showed improvement in lung function ( $\mathrm{p}=0.018$ baseline to follow up) but LC1 and LC5 showed a decline, and LC2 and LC3 showed raised eosinophils at baseline. LC1 was also associated with the $17 \mathrm{q} 21$ risk allele (OR 2.43, 95\% CI 1.07-5.53). This classification of asthma phenotypes requires external validation and its subsequent utility to inform patient management remains to be determined.

The role of eosinophils in phenotyping paediatric asthma was evaluated further by OмONy et al. [14], also using data from the ALLIANCE cohort. Children with wheeze, and children and adults with asthma were stratified into "atopy only", "raised eosinophil only", "T2 high" and "T2 low" categories. Only 6\% of children aged over 6 years showed raised eosinophils in isolation. Analysis of subcategories of age demonstrated a decline in "eosinophil only" and "T2 low" asthma from birth to adulthood before increasing again from the age of 30 years onwards. "Atopy only" asthma increased from birth until adulthood. Children aged over 6 years with the "eosinophil only" phenotype had more exacerbations within the preceding 12 months.

Continuing with asthma diagnosis, DE Jong et al. [15] used data from the Swiss Paediatric Airway Cohort (SPAC) to examine the accuracy of asthma diagnosis in children, using clinical assessment and tests 
recommended by the Global Initiative for Asthma (GINA) and National Institute for Health and Care Excellence (NICE). Of 514 participants, 69\% were diagnosed with asthma. Symptoms of "any wheeze", "wheeze triggered by colds" and "wheeze triggered by exercise" produced the highest combined sensitivity and specificity (Youden's Index). Nocturnal cough was a poor discriminator. Total specific airway resistance had the highest area under the receiver operator curve (AUC) (0.74) of any individual test, although bronchodilator reversibility was highest (0.75) when used in children with a $\mathrm{FEV}_{1} / \mathrm{FVC}$ ratio $<0.8$. Following the GINA algorithm, one-third of children would have required repeat testing to make a diagnosis. Over $80 \%$ of children would have required 2 weeks of peak-flow monitoring to make an accurate diagnosis using the NICE algorithm. The study confirms the difficulty in establishing the diagnosis in all children based on objective tests.

Finally, CAvE et al. [16] presented data on the prevalence, clinical characteristics and aspects of management of paediatric asthma in primary care in Canada. Prevalence of asthma in children aged 1-17 years was $18 \%$, with the highest prevalence (20\%) between 12 and 18 years of age. Median onset of asthma was 7 years, with males outnumbering females at all ages up to 15 years. $13 \%$ of those diagnosed required salbutamol treatment only, $66 \%$ had received inhaled corticosteroids (ICS) at some point, and $7 \%$ had received a combination ICS/LABA inhaler. Only $1 \%$ had ever received oral steroids, and only $4 \%$ had visited their doctor for asthma symptoms in the past 12 months, suggesting only a small proportion of children in primary care in Canada have severe disease.

In summary, this year's "Hot Topics in Paediatric Asthma" session explored the challenges of asthma diagnosis and attempted to define some important asthma phenotypes. This raised discussion around the utility of using the term "asthma" to represent a heterogeneous disease that may be more readily characterised and managed as a series of "treatable traits". Further work is required to characterise asthma phenotypes and, in turn, improve diagnosis and personalised management for children.

\section{Paediatric cystic fibrosis: New frontiers in CF imaging and lung physiology}

This session focussed on techniques detecting and monitoring early lung changes in cystic fibrosis. The presented studies described imaging and lung physiology results performed in children with cystic fibrosis.

BAYFIELD et al. [17] studied ultra-low-dose computed tomography in children with cystic fibrosis (aged 6-17 years). In this observational study, LCI and computed tomography were more sensitive at identifying early lung disease than spirometry and oscillometry. Despite differences in quantitative metrics, both conventional and ultra-low-dose computed tomography correlated with LCI and other MBW outcomes and showed similar sensitivity to early lung changes. Importantly, the $78 \%$ radiation reduction with ultra-low-dose computed tomography makes repeated scans a more feasible option. FRAUCHIGER et al. [18] confirmed that LCI obtained by MBW was more sensitive than $\mathrm{FEV}_{1}$ to detect early lung disease in preschool children. Instead of computed tomography, this group used functional matrix-pencil magnetic resonance imaging (MP-MRI). This radiation-free method allows assessment of structural changes and visualisation and quantification of ventilation impairment and perfusion defects. This was shown to correlate with LCI but not $\mathrm{FEV}_{1}$, demonstrating a role in early lung disease detection in pre-schoolers with cystic fibrosis.

In a second paper from the group, FRAUCHIGER et al. [19] also presented data from an observational study of LCI in 78 Swiss children with cystic fibrosis over a 7-year period. Average LCI remained stable during childhood but increased during adolescence. Risk factors associated with faster LCI increase included Pseudomonas spp. and Aspergillus fumigatus colonisation and cystic fibrosis-related diabetes. LCI also seemed to deteriorate faster in female adolescents, even in absence of specific risk factors. STANOJEvic et al. [20] studied a similar longitudinal dataset although with some differences from the Swiss study. This study followed LCI from 64 cystic fibrosis children and 50 controls from preschool into school age (to age 10 years). LCI was higher in children with cystic fibrosis, and elevated preschool LCI and greater number of cystic fibrosis exacerbations were both predictors of higher school age LCI. However, unlike the Swiss data, average LCI seemed to remain stable during these early school-age years. This may relate to subjects being younger but may also reflect the evolving nature of cystic fibrosis therapies and increased treatment intensity with cystic fibrosis transmembrane conductance regulator (CFTR) modulators and hypertonic saline. Perrem et al. [21] studied the same cohort evaluating LCI as a clinical outcome in acute respiratory events. LCI was increased and $\mathrm{FEV}_{1}$ was reduced by $10 \%$ at acute respiratory events, with an improvement when pulmonary exacerbations were treated. Recovery, however, was incomplete and had not returned to baseline by the next visit nor the next stable visit, suggesting that even milder untreated clinical events contribute to disease progression. Furthermore, LCI was also confirmed to be more responsive than FEV to untreated increased cough events and acute respiratory events.

SPANO et al. [22] addressed some practical challenges of measuring LCI in clinical practice in a third longitudinal LCI study in children with cystic fibrosis. This study showed an inverse correlation between 
$\mathrm{LCI}_{2.5}$ (LCI at $2.5 \% \mathrm{~N}_{2}$ ) and $\mathrm{FEV}_{1} \%$. For every unit increase in LCI there was a change of $-2 \% \mathrm{FEV}_{1} \%$, and for each unit increase in baseline $\mathrm{LCI}_{2.5}$, an average $-2.5 \%$ change in $\mathrm{FEV}_{1} \%$ per year. This study concluded that MBW is feasible in clinical settings but only if enough time for testing is provided.

Pennati et al. [23] used multivolume MRI ( $\left.{ }^{1} \mathrm{H}-\mathrm{MRI}\right)$ [24] as a method to assess regional intensity differences between expiratory-inspiratory scans. Following on from an earlier study [25], changes in maps of expiratory-inspiratory signal correlated with changes in $\mathrm{FEV}_{1}$ and $\mathrm{FEF}_{25-75 \%}$, indicating that multivolume MRI could be used for longitudinal evaluation of regional alterations in cystic fibrosis lung disease. This technique is radiation-free and can be carried out without inhaled or injected contrast.

Finally, Willers et al. [26] presented data demonstrating the feasibility of lung MRI in preschool children. Unsedated lung MRI was feasible in pre-schoolers but depended on age and patient anxiety.

In discussion, the value of LCI in detecting early changes was recognised and reasserted, and the practical challenges discussed. Mostly time constraints, but also lack of capacity, testing space or trained staff, lack of real-time quality-controlled results, patient adherence and boredom prevent the incorporation of this test into more routine cystic fibrosis care systems.

\section{Paediatric respiratory infection and immunology: the future of bronchiectasis management}

Bronchiectasis is the characteristic end result of a complex interaction between inflammatory, infectious, demographic and genetic factors on top of a heterogeneous spectrum of underlying aetiology [27]. The session titled "The future of bronchiectasis management" provided an overview of the bronchiectasis pathophysiology as well as recent developments in bronchiectasis management focussing on results of recent randomised-controlled trials (RCTs). Although primarily adult oriented, the topic of this symposium is also important in the context of paediatric non-cystic fibrosis bronchiectasis.

Recent evidence has emerged on the key role of mucus accumulation and its biophysical properties on pathogenesis and severity of bronchiectasis. In more detail, RAMSEY et al. [28] have demonstrated that, compared with healthy controls, bronchiectasis mucus was characterised by increased mucin concentration (primarily MUC5AC and MUC5B), higher mucus solids and higher osmotic pressure. Within bronchiectasis patients, mucus hyperconcentration was related to the extent of bronchiectasis and lower lung function while the MUC5AC/MUCC5B ratio was predictive of disease severity [28]. These results support the hypothesis of dehydrated mucus leading to inhibition of mucociliary clearance, inflammation and recurrent respiratory infections [29]. Translated into clinical practice, identification of patients with hyperconcentrated mucus, easily performed through measurement of dry-to-wet weight ratio of mucus solids, followed by therapies reducing mucus concentration, such as hypertonic saline, could provide significant benefits. Other than hypertonic saline, airway clearance techniques and mannitol have already shown to improve lung function and health-related quality of life (HR-QoL) in bronchiectasis [30-32].

The importance of the multimodality approach that should characterise bronchiectasis management, was also highlighted during the session. Rather than a linear pathophysiological pathway that could be disturbed with single treatment, bronchiectasis development is better described through a "vicious vortex" model which includes epithelial and mucus dysfunction, abnormal inflammatory response, development of structural disease and chronic recurrent infections. Based on this "vicious vortex" single treatments can only influence one component of the vortex while other components remain unaffected [33]. As a result, bronchiectasis treatment should address all aspects of the pathophysiology and include diagnosis of the underlying aetiology, assessment of disease severity, airway clearance, antibiotic and anti-inflammatory therapy [34].

Of particular interest, were the recent advances in management of chronic airway infection in bronchiectasis. Regardless of the underlying aetiology, it has been shown that chronic infection, especially with Pseudomonas aeruginosa, is associated with lower HR-QoL as well as increased hospitalisation rate and mortality [35]. A recent individual patient data meta-analysis explored the effect of macrolides on disease severity and found that macrolide treatment did not improve $\mathrm{FEV}_{1}$ but resulted in reduced exacerbation frequency (adjusted incidence rate ratio $0.49,95 \%$ CI $0.36-0.66 ; \mathrm{p}<0.001$ ) and improved HR-QoL. Furthermore, subgroup analysis demonstrated that patients benefiting the most from macrolides were older patients (>70 years), patients with high C-reactive protein and those colonised with $P$. aeruginosa [36]. The finding that $P$. aeruginosa-colonised patients respond well to macrolides contradicts the most recent ERS guidelines for long term antibiotic treatment for bronchiectasis which recommend macrolides for non- $P$. aeruginosa-colonised patients and inhaled antibiotics for $P$. aeruginosa-colonised patients [37]. Regarding inhaled antibiotics, a recent meta-analysis of 16 RCTs by LASKA et al. [38] also reported a reduction in pulmonary exacerbation ( $\mathrm{RR} 0.81,95 \%$ CI $0.67-0.97 ; \mathrm{p}=0.020$ ) and severe 
exacerbation rate ( $R R \quad 0.43,95 \%$ CI $0.24-0.78 ; \mathrm{p}=0.005$ ) although the results were characterised by significant heterogeneity and no effect was seen on quality of life. This observed heterogeneity indicates the importance of correctly selecting the patients that are most likely to respond to inhaled antibiotics. Not surprisingly, bronchiectasis patients with high bacterial load did appear to better respond to inhaled antibiotics as opposed to patient groups with lower bacterial load [39, 40]. In P. aeruginosa-colonised patients especially, inhaled antibiotics decreased pulmonary exacerbations [39]. In view of this evidence, LASKa and Chalmers [41] suggest a revised exacerbation prevention algorithm that applies in case of persistent exacerbations after macrolide treatment and recommends inhaled antibiotics for patients with high bacterial load, hypertonic saline or other mucoactive agents for patients with mucus plugging and inhaled corticosteroids for patients with eosinophilic inflammation. Lastly, directly reducing inflammation in bronchiectasis could also provide clinical benefits as brensocatib, an inhibitor of neutrophil serine proteases activation, prolonged the time to the first exacerbation and decreased exacerbation frequency in a randomised, double-blind trial in adults [42]. In regard to paediatric non-cystic fibrosis-related bronchiectasis, targeting airway inflammation has also shown to be beneficial, especially through the immunomodulatory properties of macrolides. Inhaled anti-inflammatories such as ICS, however, have not been proven beneficial $[43,44]$.

In conclusion, identifying treatable traits of bronchiectasis based on the underlying aetiology, assessing bacterial load and determining inflammatory molecular endotypes are key components for future precision medicine in bronchiectasis.

\section{Neonatology and paediatric intensive care: ventilation and chronic conditions}

Chronic morbidity related to neonatal and paediatric pulmonary disease can often pose diagnostic and therapeutic challenges. Novel research into identification of at-risk infants and children may lead to better understanding of disease pathophysiology. Development of biomarkers and genotypes may enable prediction of complications and advancement of appropriate management strategies.

Bronchopulmonary dysplasia (BPD) is a frequent and important respiratory outcome of premature birth [45] with recent work focussing on predictive models and genetic markers. Higano et al. [46] developed a tracheostomy prediction model in neonatal BPD from magnetic resonance imaging quantitative biomarkers. The model correctly classified $95 \%$ of the validation cohort and provides an objective tool for clinical decision making. Pulmonary hypertension is associated with severe BPD development [47]. HÄFNER et al. [48] showed that pulmonary arterial flow measurements taken from lung imaging are decreased in infants with BPD and correlate with days of supplemental oxygen and mechanical ventilation. This model may aid in early risk stratification of preterm infants with BPD and detect severity of lung remodelling. PAVLINOva et al. [49] related magnese superoxide dismutase (MnSOD) polymorphisms with longer-term outcomes and reported that children with BPD and the MnSOD Ala16Val genotype had a 2.4 times increased risk of adverse respiratory outcomes compared to infants with respiratory distress syndrome without BPD.

Longer term functional respiratory outcomes are important factors to consider in prematurely born infants [50]. HARRIs et al. [51] assessed the lung function at 16-19 years of age in prematurely born infants according to delivery mode at birth. Small airway function, as measured by mean $\mathrm{FEF}_{75}$, was greater in those vaginally born $(\mathrm{p}=0.009)$ suggesting the onset of labour was important in priming the early immune system. Preterm birth can also predispose infants to conditions such as patent ductus arteriosus. This may require intervention in the neonatal period, yet surgical treatment can lead to vocal cord paralysis [52]. ENGAN et al. [53] compared lung function in affected young adults at follow up compared with a control cohort and found peak oxygen consumption to be similar between the two groups ( $80 \%$ versus $79 \%$ ), yet both groups of extremely preterm infants assessed had reduced lung function and aerobic capacity irrespective of vocal cord palsy.

Impairment of oxygenation in hepatopulmonary syndrome results from intrapulmonary shunting [54]. Russell-Jones et al. [55] performed non-invasive measurements of ventilation-perfusion $\left(V^{\prime} / Q^{\prime}\right)$ indices and found a reversal of right-to-left intrapulmonary shunting post-liver transplant $(\mathrm{p}=0.009)$, yet there remained a persistence of $V^{\prime} / Q^{\prime}$ abnormalities. Changes in pulmonary dead space may reflect $V^{\prime} / Q^{\prime}$ mismatch [56]. Williams et al. [57] found the alveolar dead space to be increased in ventilated infants with evolving BPD ( $p=0.001)$, suggesting underlying pulmonary abnormalities and disease induced changes which may require ventilatory strategies with greater levels of targeted tidal volumes to allow for optimal gas exchange.

In conclusion, novel research was presented which can enable the identification of children and newborn infants at risk of longer-term respiratory morbidity, with focus on genetic and predictive biomarkers, suggesting this is a promising area for future development of pulmonary disease prevention or interventions. 


\section{Paediatric respiratory epidemiology}

Early life is a very important developmental period that may affect respiratory health throughout the life course. The results of several studies with a focus on early life exposures in relation to respiratory health were presented in this session.

LundHOLm et al. [58] used data of two large cohorts from the Swedish Medical Birth register to study the association of the airborne pollen concentration in pregnancy and infancy with current asthma in childhood. They observed that exposure to high pollen levels in infancy was related to a higher risk of asthma, whereas exposure to high pollen levels in pregnancy may protect against asthma.

Two abstracts focussed on the association of air pollution with lung function. ZHAO et al. [59] studied the association of air pollution during infancy with lung function in children of two regions in Germany aged 6 years to adolescence, and found that a higher exposure to air pollutants particles with a $50 \%$ cut-off aerodynamic diameter of $2.5 \mu \mathrm{m}\left(\mathrm{PM}_{2.5}\right), 10 \mu \mathrm{m}\left(\mathrm{PM}_{10}\right)$ and $\mathrm{NO}_{2}$ in the first year of life was associated with a -0.004 to $-0.027 \mathrm{z}$-score decline in $\mathrm{FEV}_{1}$ and $\mathrm{FVC}$ until adolescence. This association was stronger in children who were breastfed for less than 12 weeks. Similarly, LundBerg et al. [60] found in 6-month-old infants, that higher time-weighted average air pollution levels including $\mathrm{PM}_{2.5}, \mathrm{PM}_{10}$ and $\mathrm{NO}_{2}$ from birth onwards were related to a -8.39 to $-10.40 \mathrm{~mL}$ lower $\mathrm{FEV}_{0.5}$ and FVC, which are related to both obstructive and restrictive lung diseases later in life.

Two abstracts presented results on the relation of maternal diet in pregnancy with child's respiratory outcomes. Using individual participant data from seven European birth cohorts, Mensink-Bout et al. [61] studied the overall maternal diet in pregnancy and observed that a more inflammatory diet in pregnancy was related to a lower FVC in childhood, and that a very low dietary quality in pregnancy was related to an up to $20 \%$ higher risk of pre-school wheezing, a low $\mathrm{FEV}_{1} / \mathrm{FVC}$ ratio and borderline with a higher risk of school-age asthma. The study of PASHIR et al. [62] focussed on the intake of preformed vitamin A in mid-childhood in relation to lung function in adolescence, and they concluded that when comparing the top versus the bottom quartile, a higher mid-childhood intake of preformed vitamin $\mathrm{A}$, but not its provitamin $\beta$-carotene, was associated with an up to 0.18 higher $\mathrm{FEV}_{1}$ and $\mathrm{FEF}_{25-75 \%} \mathrm{z}$-score in adolescence.

Regarding antenatal growth, TuRner et al. [63] linked antenatal and birth size to the onset of asthma up to young adulthood, and mainly found that a low first trimester size as measured by crown rump length was related to earlier time of asthma admission later in life but not with asthma per se. Also, those with a low birth weight were younger when they obtained asthma medication for the first time.

The relationship of the frequency and duration of bronchitis in childhood with adverse respiratory outcomes in middle-age was studied by PERRET et al. [64], and they observed that an increase in the severity of bronchitis in childhood was associated with an increased risk of ever asthma or pneumonia, but not consistently with the risk of chronic bronchitis or impaired lung function in adulthood.

In conclusion, in this session on paediatric respiratory epidemiology, it was highlighted that early life exposure to a high pollen concentration, air pollution, a suboptimal dietary or vitamin A intake, growth restriction and bronchitis might affect respiratory health throughout the life-course.

\section{Paediatric bronchology}

We here summarise a session on paediatric procedures and several oral and poster presentations that covered many challenges and advances in paediatric bronchology. Treating complex airway pathologies within a multidisciplinary team is a new rapidly emerging approach that was reflected in many of the contributions alongside other interesting topics.

MCCARRICK et al. [65] showed the importance of multidisciplinary cooperation with paediatric ear, nose and throat (ENT) doctors in a study in which they examined 164 clinical visits of 53 children with a tracheal cannula. A flexible endoscopy was performed during 157 rounds, which showed tracheal granulomas in eight, granulomas on the stoma in 12, and both tracheal and granulomas on the stoma in two children. New recommendations regarding secretion management, tube change, fixation and skin care were made in 97 rounds. The data showed a clear improvement in patient care with a significantly lower number of procedures under general anaesthesia.

Mussel et al. [66] reported on the multidisciplinary collaboration with paediatric pneumologists, ENT and gastroenterologists in children with relevant pathologies affecting all three disciplines. The author analysed whether a combination of microlaryngoscopy, flexible bronchoscopy and oesophagogastroduodenoscopy (OGD) is beneficial. For this, they studied the medical records of 79 children over the last 3 years. The most common indication for an endoscopy was chronic cough. In 37 out of 79 children, an abnormality was found in more than one endoscopy. In six out of 20 children who received all three procedures, a 
pathology was found in all three endoscopies. The data show that different endoscopies can be performed in one setting and that the interdisciplinary exchange is thus greatly improved.

Amigo et al. [67] tackled the complex diagnosis of primary ciliary dyskinesia (PCD) by including human genetics. They designed a gene panel of 44 PCD-related genes with a sensitivity of $81 \%$ and a specificity of $100 \%$ for PCD diagnosis in 79 participants. They found 52 different gene variants, 36 not previously described in the literature. The authors showed that a specific gene panel for the genetic diagnostic of PCD is efficient and allows a better understanding of the cause of the disease, laying the basis for future therapeutic approaches.

Three abstracts on the value of lung ultrasound (LUS) were presented, underlining the close and intensive cooperation with radiology. BLOISE et al. [68] investigated the value of LUS in the diagnosis of community acquired pneumonia. They showed a very good concordance in the diagnosis of right-sided consolidations. The sensitivity in diagnosis was $97 \%$, specificity $96 \%$, sensitivity in follow-up $100 \%$, specificity $94 \%$. Delestrain et al. [69] performed LUS in five children with interstitial lung disease and compared the images to the latest computed tomography scans. In this small cohort, the authors demonstrated a good correlation between LUS and computed tomography images. Finally, LA Regina et al. [70] examined the use of LUS in bronchiolitis. They found a good correlation of the LUS score with the clinical score and with radiography. They also reported a good correlation between the findings in children with bronchiolitis and a control group.

A special highlight of the paediatric assembly was the live bronchoscopy performed by Dirk Schramm and his team. They demonstrated the importance of multidisciplinary cooperation with anaesthesiologists during interventional procedures. The patient presented suffered from juvenile-onset recurrent papillomatosis, a rare disease in children younger than 12 years caused by human papilloma virus (HPV). The mother was HPV-positive during birth and the patient has suffered from hoarseness since then. HPV type 35,45 and 87 were detected during the first bronchoscopy with bronchoalveolar lavage (BAL) at the age of 3. A course of three vaccinations with Gardasil (recombinant human papillomavirus vaccine (types $6,11,16,18)$ ) were initiated and after the second immunisation, the virus was no longer detectable in the BAL. After completion of all three vaccination cycles, a significant reduction of mucosal lesions could be seen during the live bronchoscopy, though the hoarseness remained. HPV immunisation aims at increasing the immune response and curing the disease.

To conclude, multidisciplinary cooperation is an important objective of providing exceptional patient care. In paediatric bronchology, the involvement of other disciplines such as radiology or ENT has become an integral part of clinical daily life.

\section{Lung and airway developmental biology}

This oral session covered various studies looking at effects on lung growth and alveolar development.

SELLE et al. [71] hypothesised that maternal obesity results in structural and functional changes in the lungs via alteration of adipocytokines and specifically interleukin (IL)-6. Their murine model demonstrated that maternal obesity activates IL-6 signalling in the lung of the offspring, with increased senescence, resulting in reduced alveolar type 2 (ATII) cells and decreased alveolarisation in the offspring lung. MoHr et al. [72] then continued the IL-6 story specifically looking at the impact on alveolar formation in BPD. They demonstrated that an IL-6 deficiency protects against altered alveolar formation after mechanical ventilation in BPD. This results in an upregulation of alveolar progenitor cells through a downregulation in Krüppel-like factor 4 (Klf4) and it is this in ATII that promotes alveolarisation [72]. The story was completed by HiRAni et al. [73] with their study of the role of Klf4 in myofibroblast transition and matrix remodelling after hyperoxia in BPD. They showed that downregulation in Klf4 may result from hyperoxia associated with mechanical ventilation and showed that a reduction of Klf4 promotes myofibroblast trans-differentiation and, conversely, if there is overexpression of Klf4 there is a complete block of myofibroblast trans-differentiation. Overall, they demonstrated that an overexpression of Klf4 potentially results in a pro-fibrotic phenotype with induced lung growth and matrix remodelling [73].

Moving away from hyperoxia and mechanical ventilation, WANG et al. [74] presented their study on intrauterine growth retardation (IUGR) and potential later life impacts, hypothesising that hypoxia contributes to both IUGR and deficiency in development of airway smooth muscle. Their murine model showed that reduced fetal size due to hypoxia positively correlated with airway smooth muscle thickness, and a potential predisposition to asthma.

MiziKova et al. [75] demonstrated a novel single-cell RNA analysis of the cell populations in normal and impaired late lung development. This single-cell map of the developing lung in BPD used scRNA-Seq MULTI-seq multiplex labelling of more than 66000 cells across three timepoints in 36 mice in either 21\% 
or $85 \%$ oxygen. They found that, within the endothelium, hyperoxia gradually depletes Car4 $4^{-}$cells and increases Car $4^{+}$cells. This induces inflammatory and anti-angiogenic expression in capillary endothelial cells, giving potential markers of changes induced by hyperoxia in the endothelium. Additionally, they found a depleted alveolar macrophage population and increased circulating neutrophils after hyperoxia in the second week of life [75].

WheELock et al. [76] presented data from the LUNAPRE (lung obstruction in early adulthood or prematurely born) cohort. They reported insights from the metabolomics of the cohort and current clinical status. The BPD group had reduced $\mathrm{FEV}_{1} / \mathrm{FVC}$ ratio at $18-22$ years, and a corresponding altered and unique urinary eicosanoid profile with downregulation of thromboxane and prostaglandin $\mathrm{D}_{2}$. There were also differences in profile between males and females with BPD.

BAtozhargalova et al. [77] performed a case-control study looking for potential genes predisposing to asthma in Buryat adolescents, an indigenous group from Mongolia. They identified three SNPs associated with asthma; GSDMB (rs7216389), NOS1 (AAT)n and NOS3 (VNTR). Additionally, sex differences were seen alongside a confirmation of the asthma association with SNPs in the $17 \mathrm{q} 21$ region GSDMB (rs7216389) seen in other ethnic groups [78]. Further work explored gene-gene interactions in the cohort with the GSDMB (rs7216389) SNP playing a key role.

Finally, DiAconu et al. [79] presented outcomes of a UK cohort of children treated with nusinersen for spinal muscular atrophy. 12 patients had completed loading doses of nusinersen since 2017 and while there were variable impacts on individual respiratory status, there was a $69 \%$ reduction in the number of admissions to intensive care for respiratory exacerbations.

In conclusion, it is clear from the initial presentations that early life (and fetal exposures) have subsequent impacts on the predisposition to asthma and BPD, and these mechanistic studies in conjunction with gene analysis, give us a greater insight into this. Evolving methods such as single-cell RNA analysis will open new research possibilities, while drugs such as nusinersen may have significant impacts on clinical management of complex neuromuscular diseases opening up further horizons.

\section{Concluding remarks}

The latest research presented during the ERS virtual International Congress 2020 paediatric sessions revealed key advances and concepts that will further the paediatric lung field. The importance of the identification of early life exposures that may affect long-term pulmonary health, the characterisation of phenotypes and genotypes, and the identification of biomarkers and treatable traits of different respiratory diseases was highlighted throughout the sessions. These are key elements to develop new preventive strategies and personalised management that may improve life-long respiratory health.

Support statement: C. Ardura-Garcia is supported by the Swiss National Science Foundation (grant number 32003B_162820).

Conflict of interest: C. Ardura-Garcia has nothing to disclose. S. Cuevas-Ocaña has nothing to disclose. N. Freitag has nothing to disclose. A. Kampouras has nothing to disclose. J.A. King has nothing to disclose. P. Kouis has nothing to disclose. S.M. Mensink-Bout has nothing to disclose. A.L. Whitehouse has nothing to disclose. E. Williams has nothing to disclose. T. Dassios has nothing to disclose. L. Duijts has nothing to disclose. R.H. Ersu has nothing to disclose. E.A. Gaillard reports consultancy work for Boehringer Ingelheim with money paid to his institution (University of Leicester); an investigator-led research grant from Circassia, Gilead and Chiesi Limited; and research collaboration with Medimmune. A.R. Horsley reports grants from the Cystic Fibrosis Trust and the Cystic Fibrosis Foundation; personal fees from Mylan Pharmaceuticals and Vertex Pharmaceuticals; nonfinancial support from Innovision ApS; and grants from the National Institute of Health Research, all outside the submitted work. M. Proesmans has nothing to disclose R. Rottier has nothing to disclose. D. Schramm has nothing to disclose. A. Moeller has nothing to disclose M.W. Pijnenburg has nothing to disclose.

\section{References}

1 Bårdsen T, Benestad M, Røksund $\mathrm{O}$, et al. Lung function in extremely preterm born adults over three decades. Eur Respir J 2020; 56: Suppl. 64, 3949.

2 Marsol LC, Mesa IDM, Prado OS, et al. Development of lung function in "healthy" preterm infants during the first six years of life. Eur Respir J 2020; 56: Suppl. 64, 3950.

3 Mahmoud YS, Oestreich M-A, Latzin P, et al. Does the mode of delivery influence respiratory outcomes in the first year of life? Eur Respir J 2020; 56: Suppl. 64, 3953.

4 Fouzas S, Frima ES, Theodorakopoulos I, et al. Fluctuation analysis of $\mathrm{FEV}_{1}$ in healthy children and adolescents: the effect of age. Eur Respir J 2020; 56: Suppl. 64, 3952.

5 Kentgens A-C, Lagiou O, Frauchiger BS, et al. Volumetric capnography: a promising lung function test in cystic fibrosis? Eur Respir J 2020; 56: Suppl. 64, 3954.

6 Mylemans E, Jacobs S, Vermeiren E, et al. Impact of obstructive sleep apnea and obesity on endothelial dysfunction in children. Eur Respir J 2020; 56: Suppl. 64, 3951. 
7 Hatziagorou E, Mantsiou C, Lialias I, et al. Long-term course of lung clearance index among patients with cystic fibrosis. Eur Respir J 2020; 56: Suppl. 64, 1178.

8 Macleod KA, Burns P, Marei MM, et al. Long term follow up of antenatally detected congenital lung malformation suggests lung health is not dependent on early surgery. Eur Respir J 2020; 56: Suppl. 64, 1167.

9 Eralp EE, Yegit CY, Gokdemir Y, et al. Night-to-night variability of polygraphy in children with obstructive sleep apnea. Eur Respir J 2020; 56: Suppl. 64, 1230.

10 Lelii M, Senatore L, Morello W, et al. Sleep-disordered breathing in children with chronic kidney disease: a pilot study. Eur Respir J 2020; 56: Suppl. 64, 1229.

11 Beydon N, Le Thai C, Lacin F. Cut-offs for snoring index in children suspected of obstructive sleep apnea. Eur Respir J 2020; 56: Suppl. 64, 1236

12 Sørensen KG, Øymar K, Halvorsen T, et al. Asthma, atopy and lung function in young adults hospitalised for bronchiolitis in infancy. Eur Respir J 2020; 56: Suppl. 64, 4025.

13 Hose A, Bahmer T, Rabe K, et al. Longitudinal data-driven definition of clinical asthma phenotypes in the pediatric arm of the All Age Asthma Cohort (ALLIANCE) of the German Center for Lung Research (DZL). Eur Respir J 2020; 56: Suppl. 64, 4021.

14 Omony J, Grychtol R, Maison N, et al. The role of eosinophils in pediatric and adult asthma. Eur Respir J 2020; 56: Suppl. 64, 4023.

15 de Jong CC, Pedersen ES, Mozun R, et al. Diagnosis of asthma in children: findings from the Swiss Paediatric Airway Cohort. Eur Respir J 2020; 56: Suppl. 64, 2000132.

16 Cave A, Gillies C, Aponte-Hao S, et al. Paediatric Asthma in Canada 2016: A cross-sectional study in primary care. Eur Respir J 2020; 56: Suppl. 64, 4024.

17 Bayfield KJ, Alton E, Irving S, et al. Nitrogen offset in $\mathrm{N}_{2}$ multiple washout method. ERJ Open Res 2020; 6 : 00043-2020.

18 Frauchiger BS, Willers C, Salem Y, et al. Lung clearance index and functional MRI outcomes to assess lung disease in preschool children with cystic fibrosis. Eur Respir J 2020; 56: Suppl. 64, 4311.

19 Frauchiger BS, Binggeli S, Yammine S, et al. Longitudinal course of clinically measured lung clearance index in children with cystic fibrosis. Eur Respir J 2020; 56: Suppl. 64, 4312.

20 Stanojevic S, Davis S, Sanders D, et al. Early determinants of lung disease in children with cystic fibrosis. Eur Respir J 2020; 56: Suppl. 64, 4313.

21 Perrem L, Stanojevic S, Shaw M, et al. Lung clearance index to track acute respiratory events in school-age children with cystic fibrosis. Am J Respir Crit Care Med 2020; in press [https://doi.org/10.1164/rccm.202006-2433OC].

22 Spano J, Ryan S, Milla C. Longitudinal assessment of lung clearance index as a marker of disease progression in a cohort of children with cystic fibrosis and normal spirometry. Eur Respir J 2020; 56: Suppl. 64, 4315.

23 Pennati F, Borzani I, Moroni L, et al. Multivolume magnetic resonance imaging $\left({ }^{1} \mathrm{H}\right.$-MRI) for the longitudinal assessment of cystic fibrosis (CF) lung disease: comparison with spirometry. Eur Respir J 2020; 56: Suppl. 64, 4316.

24 Pennati F, Quirk JD, Yablonskiy DA, et al. Assessment of regional lung function with multivolume ${ }^{1} \mathrm{H}$ MR imaging in health and obstructive lung disease: comparison with ${ }^{3} \mathrm{He}$ MR imaging. Radiology 2014; 273: 580-590.

25 Pennati F, Salito C, Borzani I, et al. Quantitative multivolume proton-magnetic resonance imaging in patients with cystic fibrosis lung disease: comparison with clinical indicators. Eur Respir J 2019; 53: 1702020.

26 Willers CC, Frauchiger BS, Salem Y, et al. Feasibility of unsedated lung MRI in preschoolers with cystic fibrosis a comparison to lung function. Eur Respir J 2020; 56: Suppl. 64, 4317.

27 Chalmers JD, Aliberti S, Blasi F. Management of bronchiectasis in adults. Eur Respir J 2015; 45: 1446-1462.

28 Ramsey KA, Chen AC, Radicioni G, et al. Airway mucus hyperconcentration in non-cystic fibrosis bronchiectasis Am J Respir Crit Care Med 2020; 201: 661-670.

29 Button B, Cai L-H, Ehre C, et al. A periciliary brush promotes the lung health by separating the mucus layer from airway epithelia. Science 2012; 337: 937-941.

30 Bilton D, Tino G, Barker AF, et al. Inhaled mannitol for non-cystic fibrosis bronchiectasis: a randomised, controlled trial. Thorax 2014; 69: 1073-1079.

31 Kellett F, Robert NM. Nebulised 7\% hypertonic saline improves lung function and quality of life in bronchiectasis. Respir Med 2011; 105: 1831-1835.

32 Muñoz G, de Gracia J, Buxó M, et al. Long-term benefits of airway clearance in bronchiectasis: a randomised placebo-controlled trial. Eur Respir J 2018; 51: 1701926.

33 Flume PA, Chalmers JD, Olivier KN. Advances in bronchiectasis: endotyping, genetics, microbiome, and disease heterogeneity. Lancet 2018; 392: 880-890.

34 O’Donnell AE. Medical management of bronchiectasis. J Thorac Dis 2018; 10: S3428.

35 Chalmers JD, Goeminne P, Aliberti S, et al. The bronchiectasis severity index. An international derivation and validation study. Am J Respir Crit Care Med 2014; 189: 576-585.

36 Chalmers JD, Boersma W, Lonergan M, et al. Long-term macrolide antibiotics for the treatment of bronchiectasis in adults: an individual participant data meta-analysis. Lancet Respir Med 2019; 7: 845-854.

37 Polverino E, Goeminne PC, McDonnell MJ, et al. European Respiratory Society guidelines for the management of adult bronchiectasis. Eur Respir J 2017; 50: 1700629.

38 Laska IF, Crichton ML, Shoemark A, et al. The efficacy and safety of inhaled antibiotics for the treatment of bronchiectasis in adults: a systematic review and meta-analysis. Lancet Respir Med 2019; 7: 855-869.

39 Chalmers JD, Cipolla D, Thompson B, et al. Changes in respiratory symptoms during 48-week treatment with ARD-3150 (inhaled liposomal ciprofloxacin) in bronchiectasis: results from the ORBIT-3 and-4 studies. Eur Respir J 2020; 56: Suppl. 64, 4136.

40 Sibila O, Laserna E, Shoemark A, et al. Airway bacterial load and inhaled antibiotic response in bronchiectasis Am J Respir Crit Care Med 2019; 200: 33-41.

41 Laska IF, Chalmers JD. Treatment to prevent exacerbations in bronchiectasis: macrolides as first line? Eur Respir J 2019; 54: 1901213

42 Chalmers JD, Haworth CS, Metersky ML, et al. Phase 2 Trial of the DPP-1 Inhibitor Brensocatib in Bronchiectasis. N Engl J Med 2020; 383: 2127-2137.

43 Masekela R, Green RJ. The role of macrolides in childhood non-cystic fibrosis-related bronchiectasis. Mediators Inflamm 2012; 2012: 134605. 
Pizzutto SJ, Hare KM, Uphan
Front pediatr 2017; 5: 123.

\section{Suppl. 64, 4795.} extremely premature with a history of neonatal patent ductus arteriosus surgery. Eur Respir J 2020; 56: Suppl. 64, 4793.

54 Ho V. Current concepts in the management of hepatopulmonary syndrome. Vasc Health Risk Manag 2008; 4: $1035-1041$.

55 Russell-Jones E, Dassios T, Grammatikopoulos T. Comparison of oxygen-haemoglobin dissociation curves (ODC) and $99 \mathrm{mTc}$-macroaggregated-albumin lung scans (MAA) in the diagnosis of Hepatopulmonary Syndrome (HPS) in children. Eur Respir J 2020; 56: Suppl. 64, 4792.

56 Robertson HT. Dead space: the physiology of wasted ventilation. Eur Respir J 2015; 45: 1704-1716.

57 Williams E, Dassios T, Greenough A. Physiological deadspace in ventilated infants with differing lung pathologies Eur Respir J 2020; 56: Suppl. 64, 4794.

58 Lundholm C, Almqvist C, Ekberg S, et al. Pollen exposure in pregnancy and infancy and risk of childhood asthma. Eur Respir J 2020; 56: Suppl. 64, 4981.

59 Zhao Q, Markevych I, Berdel D, et al. Early-life exposure to air pollution and lung function development into adolescence: the GINIplus/LISA birth cohorts. Eur Respir J 2020; 56: Suppl. 64, 4982.

60 Lundberg B, Gruzieva O, Eneroth K, et al. Impaired infant lung function in relation to air pollution exposure. Eur Respir J 2020; 56: Suppl. 64, 4983.

61 Mensink-Bout R, Van Meel ER, De Jongste JC, et al. Pro-inflammatory and low quality maternal diet in pregnancy and the risk of childhood lower lung function and asthma: a meta-analysis of 18,000 children. Eur Respir J 2020; 56: Suppl. 64, 4984.

62 Pashiri MT, Lietz G, Granell R, et al. Dietary vitamin A intake and lung function in childhood: longitudinal study. Eur Respir J 2020; 56: Suppl. 64, 4985.

63 Turner S, Aucott L. Reduced antenatal and birth size and time to onset of asthma. Eur Respir J 2020; 56 : 4986.

64 Perret J, Lowe A, Wurzel D, et al. Childhood bronchitis and adult respiratory outcomes: a prospective study from age 7 to 53 years. Eur Respir J 2020; 56: Suppl. 64, 4987.

65 MacCarrick T, Sharma R, Selby A. Review of a children's complex airway clinic. Eur Respir J 2020; 56: Suppl. 64, 3522.

66 Mussell G, Wall G, Thevasagayam R, et al. The use of combined endoscopy in a UK paediatric aerodigestive program. Eur Respir J 2020; 56: Suppl. 64, 5289.

67 Amigo SR, Redon NB, Tarruella NC, et al. Implementation of a gene panel for the genetic diagnosis of primary ciliary dyskinesia. Eur Respir J 2020; 56: Suppl. 64, 5293.

68 Bloise S, La Regina DP, Iovine E, et al. Lung ultrasound for the diagnosis of community-acquired pneumonia in children: comparison with chest X-ray. Eur Respir J 2020; 56: Suppl. 64, 5287.

69 Delestrain C, Thouvenin G, Richard N, et al. Lung ultrasound in children with interstitial lung disease: a pilot study. Eur Respir J 2020; 56: Suppl. 64, 5288.

70 La Regina DP, Bloise S, Nenna R, et al. Lung ultrasound (LUS) in bronchiolitis. Eur Respir J 2020; 56: Suppl. 64, 5291.

71 Selle J, Dinger K, Kesler $\mathrm{H}$, et al. Maternal and perinatal obesity arrests alveolarization and reduces alveolar progenitor cells in offspring. Eur Respir J 2020; 56: Suppl. 64, 5241.

72 Mohr J, Kesler H, Hirani D, et al. Loss of IL-6 promotes alveolar progenitor cells and lung growth in newborn mice exposed to mechanical ventilation. Eur Respir J 2020; 56: Suppl. 64, 5243.

73 Hirani D, Koch M, Mohr J, et al. Novel KLF4-FOXO1 interactome in lung fibroblasts: regulator of myofibroblasts in hyperoxia-induced lung injury. Eur Respir J 2020; 56: Suppl. 64, 5244.

74 Wang K, Noble P. Fetal weight is inversely correlated with airway smooth muscle thickness after hypoxia in mice. Eur Respir J 2020; 56: Suppl. 64, 5245.

75 Mizikova I, Hurskainen M, Cook D, et al. Single cell RNA analysis of cellular niche in normal and impaired late lung development. Eur Respir J 2020; 56: Suppl. 64, 5242.

76 Wheelock C, Meister I, Gomez C, et al. Urinary metabolomics identifies molecular signatures associated with bronchopulmonary dysplasia (BPD) and birth-term. Eur Respir J 2020; 56: Suppl. 64, 5246.

77 Batozhargalova B, Mizernitskiy Y, Petrova N, et al. Analysis of gene-gene interactions in Buryats adolescents with asthma. Eur Respir J 2020; 56: Suppl. 64, 5247.

78 Batozhargalova B, Mizernitskiy Y, Petrova N, et al. Candidate genes predisposing to the development of asthma in Buryat adolescents. Eur Respir J 2020; 56: Suppl. 64, 5249.

79 Diaconu M, Duru C, Ugonna K, et al. Respiratory outcomes pre and post nusinersen in children with spinal muscular atrophy (SMA) in a tertiary UK paediatric respiratory and neurology centre. Eur Respir J 2020; 56: Suppl. 64, 5248. 